\title{
Síndrome lumbocostovertebral. Reporte de un caso
}

\author{
Lumbocostovertebral syndrome. A case report \\ Mónica Galarza-Armijos ${ }^{1} \mathbb{D}$, Luis Marcano-Sanz² $\mathbb{D}$, Miurkis Endis-Miranda² $\mathbb{D}$, \\ Mauricio Siavichai-Romero ${ }^{2}$ (D)
}

1 Médica, residente de Cirugía General, Universidad de Cuenca, Azuay, Ecuador.

2 Médico, especialista en Cirugía Pediátrica, Unidad de Cirugía Pediátrica, Hepatobiliar y Trasplante, Hospital Vicente Corral Moscoso, Cuenca, Ecuador.

\section{Resumen}

Introducción. El síndrome lumbocostovertebral es una anomalía poco frecuente, que afecta a los cuerpos vertebrales, las costillas y músculos de la pared abdominal, y puede estar asociada a múltiples anomalías congénitas. Requiere un manejo multidisciplinario y tratamiento quirúrgico temprano para evitar complicaciones.

Caso clínico. Se reporta el caso de un neonato masculino de 10 días de edad, que presentó desde el nacimiento 2 tumoraciones lumbares derechas, una que aumentaba de tamaño con el llanto, correspondió a una hernia lumbar, y la otra, a mielomeningocele. En estudios complementarios se evidenció fusión de costillas, fusión de cuerpos vertebrales lumbares, sacros (hemivértebras) y defecto de pared abdominal con protrusión de contenido intestinal. Se realizó cierre de la hernia lumbar con refuerzo protésico de pericardio bovino, sin complicaciones.

Conclusión. En defectos extensos, como el que se reporta en este paciente, puede ser recomendable emplear material protésico. El pericardio bovino aparece como una opción segura, bien tolerada y eficaz para estos pacientes en particular. Este síndrome es una entidad rara, que amerita un equipo multidisciplinario para la resolución quirúrgica temprana y con ello evitar complicaciones.

Palabras clave: hernia; hernia abdominal; vértebras lumbares; meningomielocele; anomalías congénitas; síndrome.

\begin{abstract}
Introduction. Lumbocostovertebral syndrome is a rare anomaly that affects the vertebral bodies, ribs and muscles of the abdominal wall, and can be associated with multiple congenital abnormalities. It requires multidisciplinary management and early surgical treatment to avoid complications.
\end{abstract}

Fecha de recibido: 03/03/2021 - Fecha de aceptación: 30/04/2021 - Publicación en línea: 17/09/2021

Correspondencia: Luis Enrique Marcano Sanz, Ph.D. Hospital Regional Vicente Corral, Av. Los Arupos y Av. 12 de Abril, Cuenca, Ecuador. 010150. Teléfono: 593-998588878. Dirección electrónica: marcanosanz@gmail.com

Citar como: Galarza-Armijos M, Marcano-Sanz L, Endis-Miranda M, Siavichai-Romero M. Síndrome lumbocostovertebral. Reporte de un caso. Rev Colomb Cir. 2022;37:151-5. https://doi.org/10.30944/20117582.907

Este es un artículo de acceso abierto bajo una Licencia Creative Commons - BY-NC-ND https://creativecommons.org/licenses/by-nc-nd/4.0/deed.es 
Clinical case. The case of a 10-day-old male neonate is reported, who presented from birth 2 right lumbar tumors, one that increased in size with crying, corresponded to a lumbar hernia, and the other, to myelomeningocele. In complementary studies, rib fusion, fusion of lumbar and sacral vertebral bodies (hemivertebrae), and abdominal wall defect with protrusion of intestinal contents were evidenced. The lumbar hernia was closed with prosthetic reinforcement with a bovine pericardium, without complications.

Conclusion. In extensive defects, such as the one reported in this patient, it may be advisable to use prosthetic material. The bovine pericardium appears as a safe, well tolerated and effective option for these patients in particular. This syndrome is a rare entity, which requires a multidisciplinary team for early surgical resolution and thus avoid complications.

Keywords: hernia; abdominal hernia; lumbar vertebrae; meningomyelocele; congenital abnormalities; syndrome.

\section{Introducción}

El síndrome lumbocostovertebral (SLCV) es una enfermedad rara, que fue descrito por primera vez por Touloukian en $1972{ }^{1}$ Está conformado por hemivértebras, anomalías costales e hipoplasia de los músculos de la pared abdominal, que se manifiesta como una hernia lumbar ${ }^{1-3}$. Se puede asociar a agenesia renal, hernia diafragmática, hernia inguinal bilateral, extrofia de cloaca, hidrocefalia, atresia de ano o mielomeningocele, pie equinovarus y anomalías cardiacas, entre otras ${ }^{4-8}$.

En su primera descripcion, Touloukian refería como causa un defecto somático durante la tercera a la quinta semana gestacional ${ }^{1,3}$. Actualmente la patogenia aun es desconocida ${ }^{2}$. Existe predisposición por el sexo masculino y en algunos casos se asocia a diabetes materna ${ }^{9,10}$.

Para confirmar el diagnostico son de elección la ecografia, la tomografía computarizada (TC) y la resonancia magnética nuclear (RMN), que además dan información sobre el contenido de la hernia y el nivel de afección de las vertebras y costillas ${ }^{7,9}$.

Es importante mencionar que el SLCV amerita una resolución quirúrgica temprana debido a que, a medida que el niño crece, se hace más dificil la reparación y aumenta la posibilidad de complicaciones ${ }^{4}$. Existen reportados menos de 30 casos en la literatura consultada ${ }^{1,5}$.

\section{Caso clínico}

Masculino de 10 días de edad, peso 2320 gr, que en la ecografía 6 semanas previas a la cesárea se identificó menigocele, retardo del crecimiento in- trauterino, ectasia renal izquierda y criptorquidia. Al examen físico presentó pabellón auricular de implantación baja, cuello corto, hipertelorismo mamario, con tumoración de $6 \times 4 \mathrm{~cm}$ en región lumbar derecha, blanda, fluctuante, con mancha hipercrómica de $2 \mathrm{~mm}$ a nivel de su base, además de otra tumoración redondeada, reducible, de $4 \mathrm{x}$ $5 \mathrm{~cm}$, que incrementaba de tamaño con el llanto (figura 1), genitales con ausencia de testículos en bolsas escrotales, campotodactilia en mano derecha y genu varum bilateral.

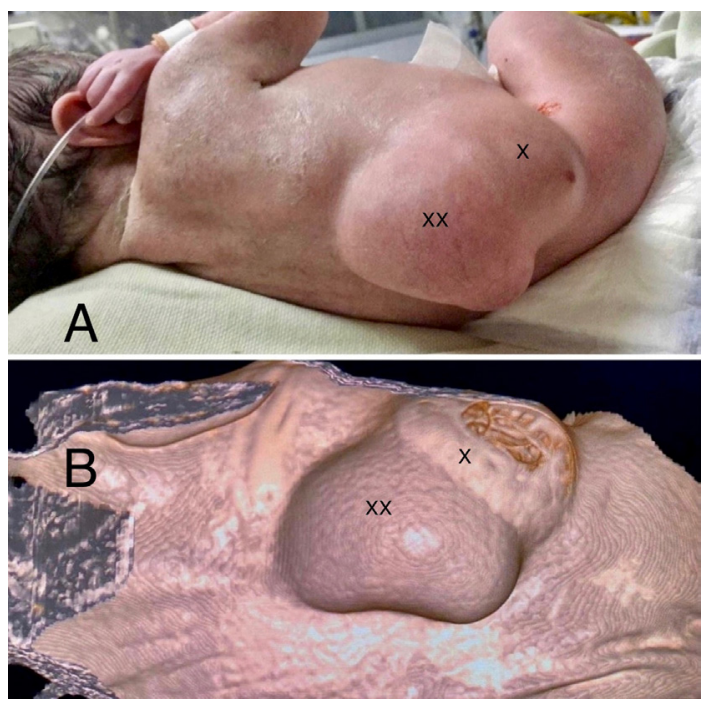

Figura 1. A: Tumoración de $6 \times 4 \mathrm{~cm}$ blanda, fluctuante, en region lumbar $(X)$ (Meningocele), y masa redondeada, reducible, de $5 \times 4 \mathrm{~cm}$, en flanco derecho,que incrementaba de tamaño con el llanto (XX) (Hernia). B: Correlación clínica con la reconstrucción de la imagen tomográfica. 
La TC de columna reportó multiples alteraciones esqueléticas del raquis y la caja toracica, fusión de las costillas derechas primera y segunda; tercera y cuarta, 11 y 12, además costilla 13 accesoria derecha y costillas 13 y 14 del lado izquierdo, así como multiples defectos de fusión de cuerpos vertebrales lumbares y sacros, escoliosis de convexidad derecha e hidronefrosis renal izquierda más megauréter (figura 2).

En la RMN se observó defecto de fusión de T12 a L2 de aproximadamente $15 \times 9 \mathrm{~mm}$, con protrusión de contenido medular y meníngeo, formación quística de intensidad similar al líquido cefaloraquídeo de aproximadamente 5,2 x 3,3 x $3,8 \mathrm{~cm}$, así como defecto de la pared abdominal con protrusión de contenido intestinal.

El paciente fue llevado a cirugía, donde se observó que el contenido del saco herniario era intestino delgado y epiplón, y se practicó cierre de hernia lumbar con sutura a puntos separados de polipropileno 3/0. Debido al tamaño grande del defecto y el déficit de las estructuras musculares

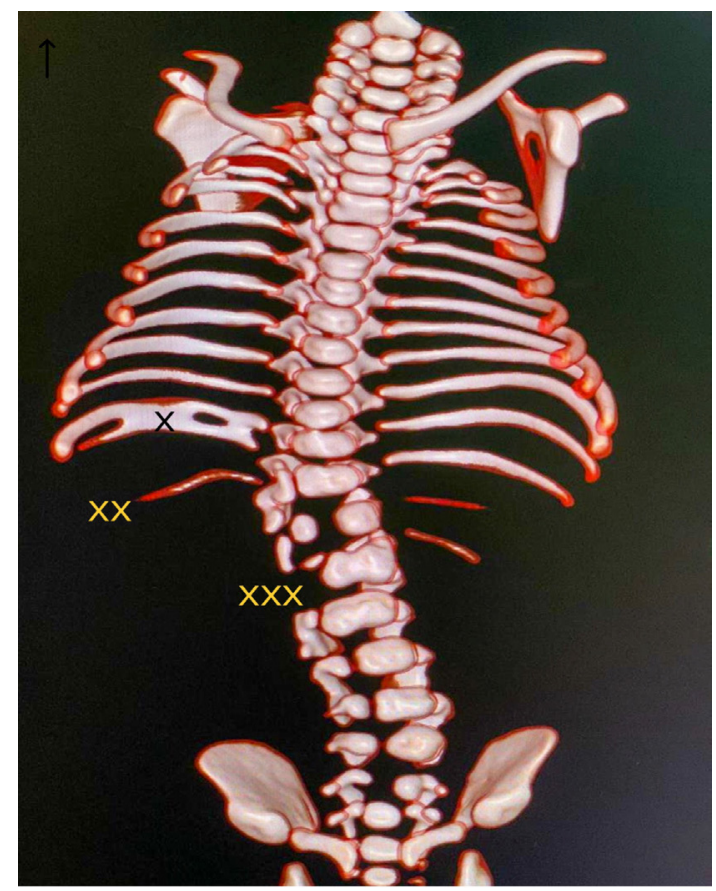

Figura 2. Tomografía computarizada de columna donde se observa fusión de costillas derechas (X), costillas accesorias $(X X)$, hemivertebras $(X X X)$ en región lumbar y sacra. de fijación, se decidió reforzar la pared con parche de pericardio de bovino, que se fijó con sutura continua de polipropileno 3-0 (figura 3). Se realizó en la misma cirugía, plastia del mielomeningocele.

El paciente evolucionó favorablemente hasta el alta, 5 días despues del procedimiento, y continuó sin signos de complicaciones en relación al parche protésico utilizado, en el seguimiento de 2 meses.

\section{Discusión}

EL SLCV es un conjunto raro de anomalías que compromete a las costillas, vertebras y músculos de la pared abdominal. La hernia lumbar es una anomalía poco frecuente que representa aproximadamente $1,5 \%$ de los defectos de la pared abdominal, es congénita en $17 \%$ de los casos y generalmente está asociada a otros defectos congénitos ${ }^{2,6}$. Se localiza en los triángulos de Grynfelt (superior), el sitio más común, y de Petit (inferior) 2,3. La hernia lumbar suele manifestarse en forma de tumoración que incrementa de tamaño durante el llanto o maniobras de Valsalva ${ }^{2,3}$.

Los cuerpos vertebrales más afectados son las últimas vértebras dorsales y las primeras lumbares ${ }^{5,6}$. Las anomalías costales consisten en

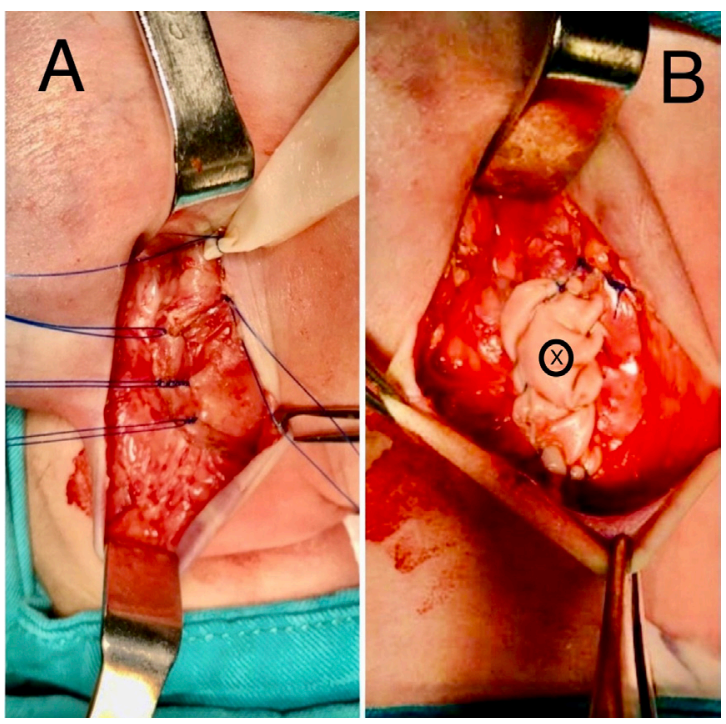

Figura 3. Aspectos de la cirugía. A: Cierre simple de hernia con puntos separados de sutura monofilamento no absorbible. B: Refuerzo con parche de pericardio de bovino $(\mathrm{X})$. 
ausencia, hipoplasia o fusión costal y las últimas costillas son las más afectadas ${ }^{6}$.

Este síndrome puede deberse a una anoxia transitoria ${ }^{1,2}$, a la alteración de la neurulación y formación defectuosa de somitas, la diferenciación en vértebras, costillas y músculos del tronco ${ }^{2,5,11}$, o alteraciones del mesodermo ${ }^{4}$. Puede estar asociado con la anomalía VACTREL, que incluye defectos vertebrales, atresia anal, defectos cardíacos, fístula traqueo-esofágica, anomalías renales, y anomalías en las extremidades ${ }^{8,12} \mathrm{o}$ también a una hernia subcostal congénita ${ }^{4}$.

El SLCV puede estar presente al nacer o notarse antes de los 2 años de edad ${ }^{2,3,9}$. El contenido del saco herniario sueler ser intestino delgado o grueso, en la mayoría de los $\operatorname{casos}{ }^{10}$, mesenterio, epiplón, apéndice, ciego, estómago, ovario, bazo en lado izquierdo ${ }^{5}$, o riñón, en raras ocasiones ${ }^{3}$. Predomina en el lado derecho $(85,7 \%)$ y en el sexo masculino ${ }^{9}$.

El tratamiento consiste en cerrar el defecto de la pared, que puede hacerse con cierre simple ${ }^{4}, 0$ refuerzo con malla de polipropilene o politetrafluoroetileno en defectos grandes ${ }^{2,3}$. La cirugía debe realizarse de forma temprana, cuando el tamaño del defecto aún es pequeño ${ }^{3}$, debido a que, el aumento de tamaño predispone a complicaciones como incarceración y estrangulación ${ }^{13}$.

\section{Conclusiones}

El Síndrome lumbocostovertebral es una entidad rara, que puede estar asociado a múltiples malformaciones congénitas, que amerita atención por un equipo multidisciplinario y resolución quirúrgica temprana para lograr una mejor calidad de vida del paciente. Es de destacar que en los defectos extensos, como el paciente que se reporta, puede ser recomendable emplear material protésico. El pericardio bovino aparece como una opción segura, bien tolerada y eficaz para estos pacientes en particular.

\section{Cumplimientos de normas éticas}

Consentimiento informado: Se obtuvo consentimiento informado de los padres del paciente para la publicación del caso y las imágenes.
Conflicto de intereses: Los autores declararon que no existen conflictos de intereses.

Fuentes de financiación: No existen fuentes externas de financiamiento.

\section{Contribución de los autores}

Concepción y diseño del estudio: Mauricio Siavichai-Romero, Miurkis Endis-Miranda, Luis Marcano-Sanz.

Adquisición de datos: Mónica Galarza-Armijos, Luis Marcano-Sanz.

Análisis e interpretación de datos: Luis Marcano-Sanz, Mauricio Siavichai-Romero, Miurkis Endis-Miranda, Mónica Galarza-Armijos.

Redacción del manuscrito: Mónica Galarza-Armijos, Luis Marcano-Sanz.

Revisión crítica: Mauricio Siavichai-Romero, Miurkis Endis-Miranda.

\section{Referencias}

1 Touloukian RJ. The lymbocostovertebral syndrome: a single somatic defect. Surgery. 1972;71:174-81.

2 Eshetu B, Mekonnen T, Berhane M. Lumbo-Costo-Vertebral Syndrome with Congenital Lumbar Hernia: Case Report. Ethiop J Health Sci. 2019;29:413-6. https://doi.org/10.4314/ejhs.v29i3.15

3 Gupta L, Mala TA, Gupta R, Malla SA. Lumbo-costo-vertebral syndrome with congenital lumbar hernia. APSP J Case Rep. 2014;5:5.

4 Muncie C, Ng C, Giles H, Blewett C. Congenital subcostal hernia in a patient with Lumbo-Costovertebral Syndrome, case report and review of the literature. J Ped Surg Case Reports. 2016;14:49-51. https://doi.org/10.1016/j.epsc.2016.09.008

5 Sengar M, Manchanda V, Mohta A, Jain V, Das S. Intercostal variant of lumbar hernia in lumbocostovertebral syndrome: our experience with 6 cases. J Ped Surg. 2011;46:1974-7.

https://doi.org/10.1016/j.jpedsurg.2011.05.010

6 Albarracín MA, Groisman B, Liascovich R, Barbero P, Bidondo MP. Síndrome lumbocostovertebral: primer reporte de caso en Argentina. Arch Argent Pediatr. 2019;117:E377-E381. http://dx.doi.org/10.5546/aap.2019.e377

7 Sinch G, Ahuja S, Kumar R, Chandra A, OjhaB, Singh C, Gupta S. Posterior spinal dysraphism with lumbocostovertebral syndrome. Br J Neurosurg. 2010;24:216-8. http://dx.doi.org/10.3109/02688690903506143 
8 Sisodiya RS, Panda SS, Ratan SK. Lumbo-costo-vertebral syndrome with inguinal hernia and other anomalies. BMJ Case Rep. 2017;2017:bcr2016216974. https://doi.org/10.1136/bcr-2016-216974

9 Rattan KN, Agarwal A, Dhiman A, Rattan A. Congenital Lumbar Hernia: A 15-Year Experience at a Single Tertiary Centre. Int J Pediatr. 2016;2016:7162475. https://doi.org/10.1155/2016/7162475

10 Okumus N, Bas AY, Demirel N, Zenciroglu A, Surmeli S, Ceylaner S. Lumbocostovertebral syndrome in an infant of a diabetic mother. Am J Med Genet A. 2010:152:1374-7. https://doi.org/10.1002/ajmg.a.33465
11 Kumar GS, Kulkarni V, Haran RP. Lumbo-costo-vertebral syndrome with posterior spinal dysraphism. Neurol India. 2005;53:351-3.

https://doi.org/10.4103/0028-3886.16943

12 Harris K, Dorn C, Bloom B. Lumbocostovertebral syndrome with associated VACTERL anomalad: a neonatal case report. J Perinatol. 2009;29:826-7. https://doi.org/10.1038/jp.2009.82

13 Staines-Orozco HS, Galindo-Campos MA. Hernia lumbar congénita y síndrome lumbocostovertebral. Informe de un caso y revisión de la literatura. Acta Pediatr Mex. 2008;29:166-8. 